\title{
Nursing strategies to support psychological adaptation in adult cancer patients: a scoping review
}

\author{
Estratégias de enfermagem para apoiar a adaptação psicológica em \\ pacientes adultos com câncer: uma revisão de escopo \\ Estrategias de enfermería para apoyar la adaptación psicológica en \\ pacientes adultos con cáncer: una revisión del alcance
}

How to cite this article:

Peixoto TASM, Peixoto NMSM, Pinto CAS, Santos CSVB. Nursing strategies to support psychological adaptation in adult cancer patients: a scoping review. Rev Esc Enferm USP. 2021;55:e03690. doi: https://doi.org/10.1590/S1980-220X2019039203690

Tiago André dos Santos Martins Peixoto ${ }^{1}$

(D) Nuno Miguel dos Santos Martins Peixoto $^{1}$

Cândida Assunção Santos Pinto ${ }^{2}$

Célia Samarina Vilaça de Brito Santos ${ }^{2}$

1 Universidade do Porto, Instituto de Ciências Biomédicas Abel Salazar, Porto, Portugal.

2 Escola Superior de Enfermagem do Porto, Porto, Portugal.

\begin{abstract}
Objective: To map the scientific evidence published in the literature about nursing strategies and intervention programs directed at supporting psychological adaptation in adult cancer patients. Method: A scoping review based on Joanna Briggs Institute Reviewers'Manual 2015 Methodology for JBI Scoping Reviews was conducted. Twelve databases were searched between 1 January 2012 and 31 January 2019. Results: From 2203 studies, 32 were included. Evidence was grouped in five subjects: procedures, outcome assessment measures, nursing feasibility, effectiveness and cost-effectiveness. The cognitive-behavioral therapy was most frequent intervention. These interventions were developed between 5 to 10 weeks, included 3 to 6 sessions and lasted up to 60 minutes. Despite a wide range of outcome measures employed, three main areas were identified, adjustment and coping; stress, anxiety and fear of recurrence; and quality of life. Conclusion: This review allowed to identify nursing strategies to support psychological adaptation in adult cancer patients, to assess vulnerabilities and difficulties related to nurse interventions and to recognize the need for further insights into the effectiveness and cost-effectiveness.
\end{abstract}

DESCRIPTORS

Neoplasms; Oncology Nursing; Adaptation, Psychological; Review.
Corresponding author:

Tiago André Peixoto

Escola de Enfermagem do Porto

Rua Dr. António Bernardino de Almeida

4200-072 - Porto, Portugal

tiago.andre.peixoto@hotmail.com
Received: $12 / 24 / 2019$

Approved: 09/21/2020 


\section{INTRODUCTION}

Scientific literature has provided conceptualizations increasingly focused on adaptive processes, showing that dealing with chronic diseases requires adaptation in multiple life domains ${ }^{(1-2)}$. The interest in recognizing physiological, emotional, social, behavioral and cognitive aspects of psychological adaptation to oncological disease has also grown over the last decade ${ }^{(3-4)}$.

Historically, multiple studies have been developed with the purpose of understanding the adaptive processes and relationship between stress, coping and psychological adjustment concepts ${ }^{(5-17)}$.

Consciously or unconsciously, individuals with cancer define a trajectory to go through and deal with the challenges of a chronic disease. Facing an oncological disease - with its associated physical, psychological and social integrity challenges, strong feelings of loss and suffering and vulnerability emergence - has a significant impact on how individuals define themselves and the environment around them ${ }^{(18-21)}$. Therefore, in addition to all the biological changes associated with the process of cell growth and division, cancer entails a psychosocial adaptation of the individual to his new condition ${ }^{(21-22)}$.

Adjustment or adaptation, despite often referred to as synonyms, are different concepts. Theoretically, the first integrates the process of interaction between the individual and surrounding environment and the individual response to eliminate stimuli in action at that moment; the second relates to results and implies a structural, perceptional or behavioural change by which an organism increases its chances of surviving or of answering more favourably to the environment and its conditions s $^{(1,3,8,23-25)}$.

Coping strategies present as a range of cognitive and behavioural efforts (health status acceptance, role performance, physical impairment adaptation, health status satisfaction, hope, body image, family and social support, anxiety self-control, fear of recurrence, self-esteem, disease management, sexual performance, motivation, among others) that the individual adopts to adjust to internal and external requirements emerging in stressful situations, and can be defined as a personal burden or a demand which overcomes the subject's personal resources ${ }^{(9,26)}$. Coping is not an isolated phenomenon but an integral part of a complex and dynamic process involving the subject, the environment, and the relationship between both. It is believed that coping strategies can contribute to adaptive behaviours by the individual and are able to mediate the whole process ${ }^{(27)}$.

Considering cancer acceptance, coping as an adaptive mechanism aims to solve problems related to the disease and its consequences in a realistic way, reduce feelings of threat, preserve physical integrity, help to maintain social relationships and performance in different roles, promote a positive self-concept, create a personal concept of value and social acceptance, increase self-esteem and increase quality of life (QoL) $)^{(28-33)}$.

The authors of the present work proposed to develop an educational nursing intervention able to foster the adaptive process of cancer patients following recommendations of the Medical Research Council for development and evaluation of complex interventions ${ }^{(34)}$. This study represents the first step for achieving that goal and aims to map the scientific evidence published in the literature about nursing strategies and intervention programs directed at supporting psychological adaptation in adult cancer patients.

The research question for this review was: "What is known in the existing scientific literature about nursing interventions strategies and intervention programs directed at supporting psychological adaptation in adult cancer patients?". For the construction of this question, the PPC mnemonic was used (Population - adult cancer patients, Concept - psychological adaptation, Context - nursing interventions and intervention programs). The aims, inclusion criteria and methodological approach of this review were detailed in a previously developed protocol.

\section{METHOD}

\section{Study DESIGN}

A scoping review was conducted using the methodological framework developed by Joanna Briggs Institute Reviewer's Manual for Scoping Reviews ${ }^{(35)}$ and Preferred Reporting Items for Systematic reviews and Meta-Analyses extension for Scoping Reviews (PRISMA-ScR) Checklist for data organization ${ }^{(36)}$. This type of study allows for a systematic approach to the existing scope of evidence about a specific theme, by summarizing and outlining findings, identifying knowledge gaps and suggesting future studies ${ }^{(35)}$. Therefore, this study consists of a scoping review about nursing interventions aiming at promoting a psychological adaptation to oncological disease in adults.

\section{DATA COLLECTION}

Study search for this review was accomplished by searching Academic Search Complete ${ }^{\circledR}$, Business Source Complete $^{\circledR}$, CINAHL Complete $^{\circledR}$, Educational Resource Information Center ${ }^{\circledR}$, Library, Information Science \& Technology Abstracts ${ }^{\circledR}$, MedicLatina ${ }^{\circledR}$, MEDLINE with Full Text ${ }^{\circledR}$, Psychology \& Behavioral Sciences Collection ${ }^{\circledR}$, Regional Business News ${ }^{\circledR}$, SPORTDiscus with Full Text ${ }^{\circledR}$ and Cochrane Library ${ }^{\circledR}$, using Medical Subject Heading $(\mathrm{MeSH})$ terms and free text words combined with boolean operators "OR" and "AND” and the tool “*”. Exploratory searches were previously conducted on the referred databases using the terms cancer, adaptation and nursing, so as to provide the best framework for the review. After reading and analysing some relevant articles, the most common keywords were identified. We believe that the set of selected databases is sufficiently large and, at the same time, sufficiently specific to answer the research question.

Through EBSCOhost $\mathrm{Web}^{\circledR}$ search engine, a search was conducted at Academic Search Complete ${ }^{\circledR}$, Business Source Complete ${ }^{\circledR}$, CINAHL Complete ${ }^{\circledR}$, Educational Resource Information Center ${ }^{\circledR}$, Library, Information Science \& Technology Abstracts ${ }^{\circledR}$, MedicLatina ${ }^{\circledR}$, 
MEDLINE with Full Text ${ }^{\circledR}$, Psychology \& Behavioral Sciences Collection ${ }^{\circledR}$, Regional Business News ${ }^{\circledR}$ and SPORTDiscus with Full Text ${ }^{\circledR}$ using the following boolean phrase: (((("Neoplasms") OR ("Cancer") OR ("Cancer Patient")) NOT ("Child"))) AND (("Adaptation, Psychological”) OR ("Coping”)) AND (("Nursing”) OR ("Nursing care"))). The following filters were activated: Published Date - 2012/01/01-2019/01/31.

Subsequently, Cochrane Library ${ }^{\circledR}$ was used to complete the search by using a boolean phrase specific to this database: (Neoplasms OR Cancer OR Cancer Patient NOT Child) AND (Adaptation, Psychological OR Coping) AND (Nursing OR Nursing care); Publication from 2012/01/01 to 2019/01/31; Search all text; Word variations have been searched.

This search was carried out in February 2019 and the use of some filters in the search allowed to access the most recent research on the subject, to extend the search to the full text of the articles and to consider some variations of the searched keywords. This option reduced the possibility of eliminating articles that could be considered relevant.

\section{SELECTION CRITERIA}

The search was conducted in English and scientific articles written in English, Spanish or Portuguese and published between 1 January 2012 and 31 January 2019 were eligible for inclusion. No restrictions were made concerning the type of study. Due to the high specificity of interventions directed at children and adolescents, studies including these specific patient populations were excluded, as well as those concerning interventions not amenable to be performed by nurses. Emphasizing the need to consider family and caregivers throughout the cancer journey as useful resources for nursing interventions, studies addressing both patients and partners/caregivers were also included. Studies including only partners or caregivers of cancer patients were excluded. Studies addressing the impact of nursing interventions in the process of psychological adaptation to oncological disease including nurses in the study sample were also included, due to their relevance. All disease stages (including advanced and palliative) and a comprehensive number of cancer types were considered.

Grey literature was also incorporated in the review, by including studies retrieved from free searches complying with predefined inclusion criteria. Study references were also analysed to identify further studies for inclusion. Both study review and data extraction were conducted by two independent investigators. Disagreements between the two authors were resolved by listening a third member of the research team.

\section{DATA ANALYSIS AND TREATMENT}

Retrieved studies were analyzed in two phases. On the first phase, a descriptive analysis exploring studies' methodological characteristics (study design, cancer type/ stage and sample) and results (including study year and geographic distribution) was performed. On the second phase, retrieved evidence was sorted in five groups related to nursing interventions: procedures (including the conceptual framework), outcome assessment measures, feasibility, effectiveness and cost effectiveness. Evidence tables were developed to organize data, according to the five categories above.

\section{RESULTS}

The original search retrieved 2203 studies, 576 of which from Academic Search Complete ${ }^{\circledR}, 6$ from Business Source Complete ${ }^{\circledR}, 399$ from CINAHL Complete ${ }^{\circledR}, 1$ from Library, Information Science \& Technology Abstracts ${ }^{\circledR}, 2$ from MedicLatina ${ }^{\circledR}, 943$ from MEDLINE with Full Text ${ }^{\circledR}$, 57 from Psychology \& Behavioral Sciences Collection ${ }^{\circledR}$, 1 from Regional Business News ${ }^{\circledR}, 6$ SPORTDiscus with Full Text ${ }^{\circledR}, 190$ from Cochrane Library ${ }^{\circledR}$ and 22 from the grey literature. A total of 395 were duplicates and therefore removed through a reference manager $\left(\right.$ Zotero $\left.^{\circledR}\right)$. Thirty-two articles were selected for data extraction and analysis. Figure 1 depicts the process of selection, with article selection flow from identification to final inclusion.

Most studies included in this review were published in 2013 ( $n=14)$, five in 2014, four in 2016. The scientific community interest in this theme was perceived as higher in Europe $(n=11)$ and in the American continent $(n=12)$, where five of eleven studies were retrieved from the United Kingdom and ten of twelve studies from the U.S., respectively. Studies were also included from Oceania ( $n=5$; including four from Australia) and Asia ( $n=3)$. Only two studies were from Portugal. Countries like New Zealand, Canada, Japan, Mexico, China, Singapore, Ireland, Netherlands, German and Norway contributed with one study each.

Methodological characteristics were well balanced among studies, with 11 experimental studies (including eight Randomised Controlled Trials [RCTs] and three Clinical trials with pre- and post-intervention measurements) and ten literature reviews included. Furthermore, two Quasi-experimental studies, two Pilot studies of Randomized controlled trials and three Protocols of Randomised Controlled Trials were also included. Notably, empirical studies accounted for more than $55 \%$ of analysed articles $(n=18)$.

Breast cancer was the most studied cancer type $(n=11)$. However, a relevant number of studies $(n=11)$ included more than one type of tumour simultaneously. Prostate $(n=3)$, lung $(n=2)$, head and neck $(n=2)$, hematological $(n=1)$, colorectal $(n=1)$ and gynaecological $(n=1)$ cancers were the remaining cancer types included in this analysis. Importantly, six studies included both oncological patients and their partners $(n=5)$ or caregivers $(n=1)$ and one study comprised health professionals as part of the sample, for the purpose of developing nursing interventions directed at promoting breast cancer psychological adaptation.

With the exception of one study considering the advanced/palliative setting, the concept of adaptation is mentioned in the literature in every single stage of the oncological disease. Eleven studies were found which approached adaptation independently of disease stage, eleven studies 
focused treatment stage and ten other studies focused survival/post-treatment stage.

Most studies ( $n=12)$ included samples with no less than 100 participants, three included samples with 50-100 participants and the remaining included less than 50 participants.
The subject of the present review is relevant, as it allows to understand how other intervention programs were developed and discloses procedures implemented in nursing interventions $^{(34)}$. Chart 1 summarizes the main characteristics (aims, results and conclusions) of studies included.

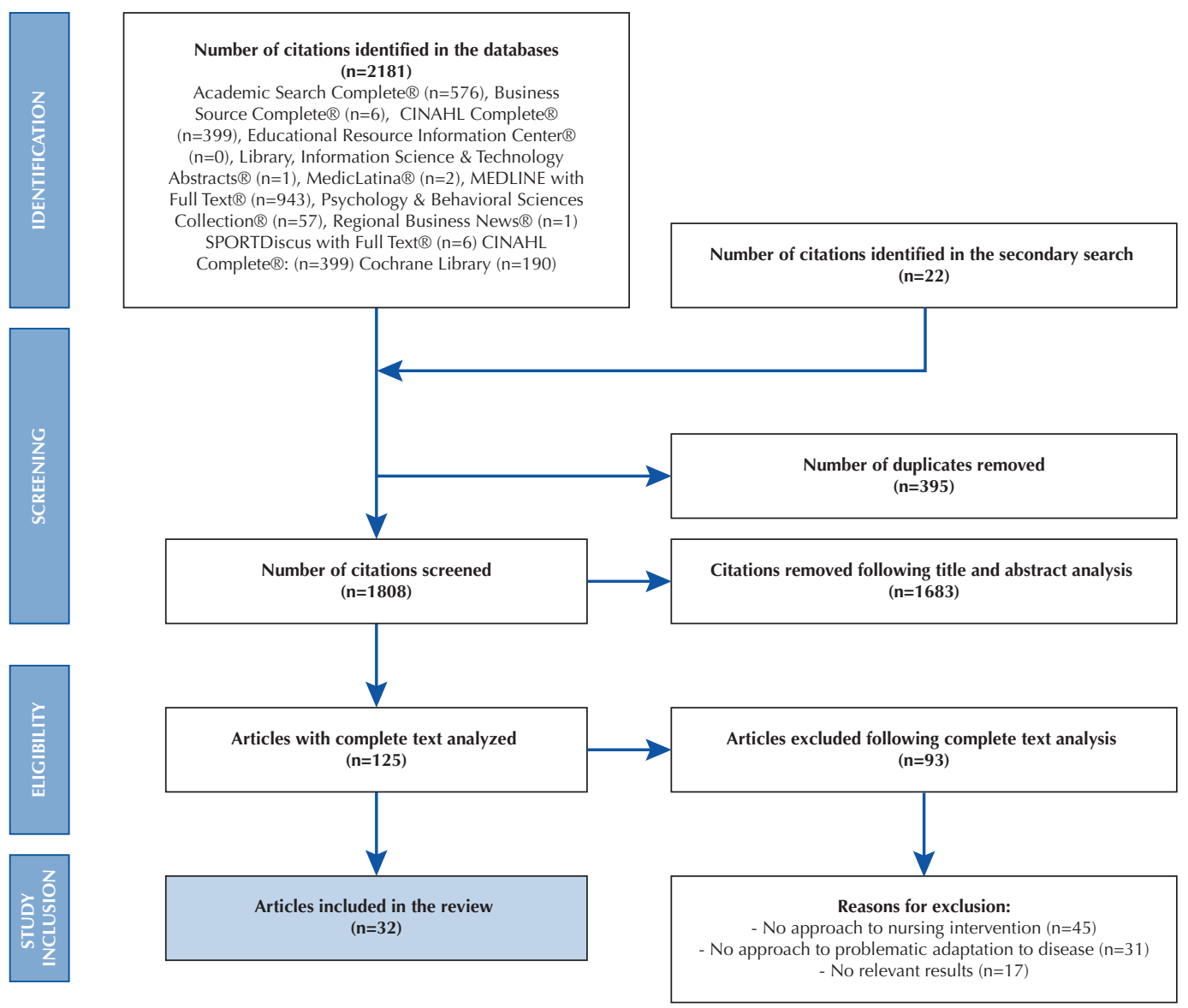

Figure 1 - PRISMA diagram flow chart of study identification and inclusion ${ }^{(36)}$.

Chart 1 - Characteristics of studies included.

\begin{tabular}{|c|c|}
\hline Author/s and Aim & Results and Conclusions \\
\hline $\begin{array}{l}\text { Decker et al. }{ }^{(37)} \text { - To develop and evaluate the feasibility } \\
\text { of a brief intervention to attenuate the incidence of } \\
\text { psychosexual morbidity within the dyad secondary to the } \\
\text { diagnosis and treatment of breast cancer. }\end{array}$ & $\begin{array}{l}\text { Interesting trends concerning differences between the intervention and comparison } \\
\text { groups on the dyadic adjustment were obtained. The intervention was feasible and } \\
\text { acceptable. Intervention by telephone was shown to be as effective as face-to-face } \\
\text { mode of delivery. }\end{array}$ \\
\hline $\begin{array}{l}\text { Lambert et al. }{ }^{(38)} \text { - To test the efficacy of Coping-Together: } \\
\text { a multimedia, self-directed, coping skills training } \\
\text { intervention for patients with cancer and their partners. }\end{array}$ & Not applicable. No results. Protocol study. \\
\hline $\begin{array}{l}\text { Lambert et al. }{ }^{(39)} \text { - To examine acceptability of methods } \\
\text { used to evaluate Coping-Together, one of the first self- } \\
\text { directed coping skill intervention for couples facing } \\
\text { cancer, and to collect preliminary efficacy data. }\end{array}$ & $\begin{array}{l}\text { For patients, results suggested that Coping-Together may improve distress (intrusion } \\
\text { and avoidance) and contribute to potentially appraising cancer as less challenging. } \\
\text { Although both groups reported a consensus decrease, Coping-Together couples } \\
\text { decline was lower than minimal ethical care (MEC) participants. Some improvements } \\
\text { in the MEC group were also noted in terms of mental quality of life, uncertainty, } \\
\text { harm/loss appraisal, and supportive dyadic coping by oneself. }\end{array}$ \\
\hline $\begin{array}{l}\text { McCaughan et al. }{ }^{(40)} \text { - To describe development } \\
\text { and evaluation of the content of a self-management } \\
\text { psychosocial intervention for men with prostate cancer } \\
\text { and their partners. }\end{array}$ & Not applicable. No results. Protocol study. \\
\hline $\begin{array}{l}\text { Chambers et al. }{ }^{(41)} \text { - To compare the effectiveness of } \\
\text { two low-intensity approaches for distressed patients with } \\
\text { cancer and caregivers who called cancer helplines seeking } \\
\text { for support. }\end{array}$ & $\begin{array}{c}\text { For low-education patients, only the psychologist intervention was associated with } \\
\text { a significant distress drop. For all other participants, distress decreased over time in } \\
\text { both arms with small to large effect sizes. Post-traumatic growth increased over time } \\
\text { for all participants. }\end{array}$ \\
\hline
\end{tabular}

continue... 
...continuation

Author/s and Aim

Results and Conclusions

Isardas et al. ${ }^{(42)}$ - Not applicable. No aim. Evidence-based care sheet (recommendations).
Not applicable. No results. Evidence-based care sheet (recommendations).
Dorros et al. ${ }^{(43)}$ - To identify primary concerns of dyads coping with cancer, how they differ by role and sex and whether concerns expressed during counselling are associated with survivors' psychosocial well-being and adjustment.

Von et al. ${ }^{(44)}$ - To provide a comprehensive summary of coping strategies and evidence-based interventions used to address cognitive impairment following cancer and cancer treatment.

Horii et al. ${ }^{(45)}$ - To retrieve basic data about support for life adjustment in lung cancer patients in Japan.
Survivors' concerns focused on cancer and treatment-related issues, whereas partners' concerns centered on the spouse/partner with cancer well-being, and what they were doing to help their loved one cope with his/her illness. Key concerns for all consisted of relationship maintenance and communication issues. Further discussion of these concerns was predictive of significant improvements in postcounselling adjustment for women with breast cancer. Discussion of interpersonal concerns may play a more important role in the well-being of women, rather than men, coping with cancer.

Survivors identified a structured environment and validation of their cognitive concerns as essential to adjustment. Although interventional research is still limited, non-pharmacological approaches such as cognitive training programs show the greatest promise.

Although requiring further testing, findings suggested that improvements in lung cancer patients' quality of life can be achieved by combining the use of this model with positive self-evaluation and interventions to raise self-adjustment ability.
Sekse et al. ${ }^{(46)}$ - To provide insights about women's self-experience in participating in an education and counselling group intervention after curative treatment for gynaecological cancer.

Main findings indicated that participation in the rehabilitation group was considered a special community moment of mutual understanding and belonging. Education and knowledge sharing endowed women with a clearer vocabulary and a better understanding of women's self-lived experiences. Attending a nurse-led education and counselling group intervention had a positive impact on various aspects of women's experiences.

Chen et al. ${ }^{(47)}$ - To assess couple-, compared with individual-based coping intervention on self-efficacy and quality of life in patients with resected lung cancer.
Couple coping intervention is more effective than individual coping intervention for improving self-efficacy and quality of life in patients with resected lung cancer.

Jefford et al. ${ }^{(48)}$ - To examine the effectiveness of an innovative supportive care program (SurvivorCare) comprising survivorship educational materials, provision of a tailored survivorship care plan (SCP), an individually tailored nurse-led end-of-treatment consultation and telephone follow-up for people with potentially cured colorectal cancer, aiming to reduce psychological distress and unmet needs in the six months following treatment completion.

Not applicable. No results. Protocol study.
Smith et al. ${ }^{(49)}$ - Pillars4Life is an educational program that teaches coping skills to cancer patients in a virtual group setting. The cost-effective, scalable, and assessable Pillars4Life curriculum targets psychosocial resources as a way to reduce symptoms and enhance quality of life.

Whitehead et al. (50) - To assess the effects of home-based maintaining or improving quality of life in breast cancer survivors.

Gaston-Johansson et al. ${ }^{(51)}$ - To examine effectiveness of a self-management multimodal comprehensive coping strategy program (CCSP) on quality of life (QoL) among breast cancer patients 1 year after treatment. multidimensional survivorship (HBMS) programmes on

At 3- and 6-month follow-up, mean scores improved for all key outcome measures, including depression, anxiety, posttraumatic stress, fatigue, and well-being from baseline. Results were most pronounced among participants who reported $\geq 4 / 10$ on the Distress Thermometer at baseline. Changes in each targeted resource were associated with 3-month improvements in, at least, one outcome. Participation in the Pillars4Life program was associated with statistical and clinically significant improvements in scores on pre-specified outcomes and targeted resources.

Systematic review and meta-analysis of randomized controlled trials (RCTs) and quasi-RCTs assessing the effects of HBMS programmes in maintaining or improving quality of life in women with stages 0 to 3 breast cancer who completed primary cancer treatment up to 10 years earlier. A total of 22 RCTs and four quasi-RCTs comprising 2272 participants were included. Intervention components were categorised into four groups: educational and psychological; educational and physical; physical and psychological; and educational, physical and psychological. Results revealed that HBMS programmes seem to have a short-term beneficial effect in improving breast cancer global quality of life in survivors. In addition, HBMS programmes were associated with anxiety, fatigue and insomnia reduction immediately after the intervention.

A model measuring effectiveness of CCSP on QoL at 1-year follow-up showed that the CCSP group significantly improved overall QoL, health and functioning, and socioeconomic and psycho-logical/spiritual well-being compared with the control group. CCSP patients frequently used the CCSP to manage psychological and sleep problems. CCSP improved QoL for patients at 1-year follow-up. Patients overwhelmingly reported that CCSP was beneficial. CCSP showed potential as a selfmanagement program for breast cancer survivors.

Thomas et al. ${ }^{(52)}$ - To describe the key components of meaning-centered psychotherapy, summarize current research pertaining to this therapy and offer suggestions for future directions of this intervention.
Meaning-centered psychotherapy appeared to effectively enhance meaning and spiritual well-being for cancer patients. Authors showed that psychotherapy can be offered in group or individual format and modified for patients with advanced cancer, cancer survivors, or even nursing staff. The longer-term benefits of treatment, its impact on emotional or physical distress, its level of cost-effectiveness, and its potential for dissemination remained unclear. 
...continuation

Author/s and Aim Results and Conclusions

Scott et al. ${ }^{(53)}$ - To conduct a systematic review of studies examining effectiveness of multidimensional rehabilitation programmes (MDRP) in maintaining or improving the physical and psychosocial well-being of adult cancer survivors.

de Leeuw et al ${ }^{(54)}$ - To compare conventional medical follow-up with follow-up including additional nursing consultations regarding psychosocial adjustment and health-related quality of life (HRQoL) of head and neck cancer patients.
Participating in a MDRP was associated with an increase in physical health component scores. Findings from the narrative analysis suggested that MDRPs with a single domain or outcome focus appeared to be more successful than programmes with multiple aims. In addition, programmes that comprised participants with different types of cancer compared to cancer site-specific programmes were more likely to show positive improvements in physical outcomes. The most effective mode of service delivery appeared to be face-to-face contact supplemented with at least one follow-up telephone call. There was no evidence that MDRPs which lasted longer than six months improved outcomes beyond the level attained at six months. There was also no evidence that services were more effective if delivered by a particular type of health professional.

Intervention group had a larger score improvement, which was significant for one of the seven adjustment scales and 19 of the $33 \mathrm{HRQoL}$ scales at 6 and 12 months, respectively. These results suggested that nurse-led consultations for patients with head and neck cancer have a positive effect, primarily concerning HRQoL. Nurseled follow-up leads to a similar psychosocial adjustment as conventional follow-up, even among patients with worse performance at follow-up start. Authors concluded that nurse-led follow-up may be a cost-effective way to improve follow-up care for this patient population.

Ten RCTs was included, seven of which on group psychological interventions, three on cognitive behavioural therapy and four on supportive-expressive group therapy. The remaining three studies were individual-based and types of psychological interventions were not common to either cognitive behavioural or supportive-expressive therapy. The overall effect of psychological interventions across six studies on one-year survival favoured the psychological intervention group. Pooled data from four studies did not show any survival benefit at five-year follow-up. There was evidence of a short-term benefit for some psychological outcomes and pain score improvements. Psychological interventions appeared to be effective in improving survival at 12 months but not at longer-term follow-up and were effective in reducing psychological symptoms only in some assessed outcomes in women with metastatic breast cancer.

A total of 28 studies were included. A wide range of interventions were evaluated with 24 trials investigating a cognitive behavioural therapy and four trials investigating psychotherapy compared to controls. A psychological intervention, namely cognitive behavioural therapy, produced favourable effects on some psychological outcomes,

particularly anxiety, depression and mood disturbance. However, evidence for a survival improvement was still lacking. The authors emphasise that study findings are open to criticism due to the notable heterogeneity and shortcomings of studies included.

Nineteen studies comparing psychosocial interventions versus usual care in a total of 3204 men with prostate cancer were included in this review. Results showed that psychosocial interventions result in small improvements in QoL at the end of intervention, although it was not clear whether they actually made a difference to participants. Interventions were also effective in increasing knowledge up to three months after the intervention. No evidence suggested that these interventions improved self-efficacy or reduced uncertainty, distress and depression in men with prostate cancer. No clear evidence showed that group-based were more effective than individual-based interventions for most outcomes measured in this review.

Seven trials, accounting for 542 participants, met eligibility criteria. Analysis of these studies showed no evidence that psychosocial interventions promotes global quality of life for patients with head and neck cancer at the end of intervention as measured by the European Organisation for Research and Treatment of Cancer Quality of Life

Questionnaire. There was no significant change in levels of anxiety or depression

following intervention. Authors concluded that, at present, there is insufficient evidence to refute or support the effectiveness of psychosocial interventions for patients with head and neck cancer.

Northouse et al. ${ }^{(59)}$ - To determine whether patientcaregiver dyads, randomly assigned to either a brief or extensive dyadic intervention (FOCUS Program), had better intermediary outcomes (i.e. less negative appraisals and increased resources) and better primary outcomes (i.e. improved QOL) than control dyads receiving only usual care; and to determine whether risk for distress and other antecedent factors (e.g. gender, type of dyadic relationship and cancer type) moderated effect of the brief or extensive program on intermediary and primary outcomes.

Sherman et al. ${ }^{(60)}$ - To examine physical, emotional and social adjustment of women with early-stage breast cancer who received psychoeducation by videotapes, telephone counseling or psychoeducation plus telephone counseling as interventions addressing women's specific needs during diagnostic, postsurgery, adjuvant therapy, and ongoing recovery phases of breast cancer.
Significant group by time interactions showed that there was an improvement in dyads' coping, self-efficacy, and social QoL, as well as in caregivers' emotional QoL. Effects varied by intervention dose. Most effects were found at 3 months only. Risk for distress accounted for very few moderation effects. Both brief and extensive programs had positive outcomes for patient-caregiver dyads, but few sustained effects.

Improvements were observed for patients in all groups concerning overall health, psychological well-being and social adjustment. No significant group differences were observed in physical adjustment, as measured by side effect incidence and severity or overall health. Poorer emotional adjustment over time was registered in the usual care (control) compared to the intervention groups concerning distress side effect. Telephone counseling group showed a marked decline in psychological wellbeing from the adjuvant therapy phase through the ongoing recovery phase. There were no significant group differences in t social adjustment dimensions.

continue... 
..continuation

Antoni ${ }^{(4)}$ - To summarize current knowledge on psychological and physiological adaptation processes affected by cancer-related stress, and the effects of psychosocial interventions on psychological adaptation, cancer disease progression, and changes in stress-related biobehavioural processes that may mediate intervention effects on clinical cancer outcomes.

According to authors, behavioural research suggests the importance of cognitive, behavioural, and social factors in facilitating adaptation during active treatment and throughout cancer survivorship, which forms the rationale for the use of many psychosocial interventions in cancer patients. Changes in adaptation may alter tumo growth-promoting processes (increased angiogenesis, migration and invasion and inflammation) and tumor defense processes (decreased cellular immunity) relevant for cancer progression and QoL of cancer patients. Some evidence suggests that psychosocial interventions can improve psychological and physiological adaptation indicators in cancer patients. Available evidence shows the ability of many different psychosocial interventions in improving stress and adversity responses to cancer experience as a way to improve psychological adaptation. Improvements in psychological adaptation (decreased negative affect and social disruption and increased positive affect and QoL) have been linked to an improved physiological profile during and after treatment, which may increase the odds for disease-free survival in some cancers.

Salgado ${ }^{(61)}$ - To contribute to the development of a set of nursing interventions promoting breast cancer adaptation as a way to improve patients' QoL.
A total of 79 nursing interventions for women with breast cancer were suggested, which remain to be tested in the clinical practice for clinical efficacy assessment.

Significant effects were found for cancer distress, global QoL, physical function, role function, social function and anxious preoccupation. Post hoc between-group comparisons showed that CCO participants had statistically significantly higher physical functioning compared to controls at 3-month follow-up. Furthermore, compared to controls, post hoc comparisons found a moderate between-group size effect favouring CCO post-interventions for cancer distress and anxious preoccupation, and at 6 months of follow-up for global QoL.
García et al. ${ }^{(63)}$ - To demonstrate the efficacy of cognitivebehavioral therapy in a sample of patients diagnosed with breast cancer in anxiety and depression reduction and in developing positive coping skills to improve QoL perception

David et al. ${ }^{(64)}$ - To test the effectiveness of an internetbased program for hematologic cancer patients tested in a randomized controlled trial under field experimental conditions

Carpenter et al. ${ }^{(65)}$ - To develop an interactive web-based version of a Cognitive Behavioral Stress Management (CBSM) intervention for breast cancer survivors - the Coping with Cancer Workbook - and to evaluate its effectiveness in a randomized waitlist-controlled trial.

Brandão et al. ${ }^{(66)}$ - To identify and describe psychological interventions for couples coping with breast cancer and evaluate their efficacy and satisfaction of individual interventions to reduce fear of cancer recurrence.
Patients who participated in the individual intervention had statistically significant differences in QoL, physical health, interpersonal relations, anxiety subscale and Hospital Anxiety and Depression Scale (HADS). Authors concluded that cognitivebehavioral therapy was efficacious in the sample studied.

At registration, most participants displayed clinically significant distress and lacked alternative access concerning psychosocial care. In contrast to the waiting list, the intervention group displayed a significant increase in fighting spirit. The effect was confirmed by intention-to-treat analysis. Otherwise, no effects were observed. Patient satisfaction with the program was high. Results demonstrated the potential efficacy of internet-based programs, also highlighting their limitations.

An internet intervention based on an empirically supported CBSM intervention appeared to be effective in helping breast cancer patients live with more confidence in their ability to cope with stress. Given the increasing demands on healthcare and the fact that breast cancer patients are living longer as survivors, it is critical to find low impact ways to improve their lives. More research is needed to ascertain the long-term effects of such interventions, whether women are able to maintain these positive changes, and whether such an intervention can help prevent incidence of depression and other disorders in cancer patients.

Ten studies were included. Conflicting results were obtained regarding efficacy, although the large majority of studies $(n=8)$ found benefits for both women and their

partners in some dimensions, including QoL, psychological distress, relationship functioning, and physical symptoms associated with cancer. Psychological interventions

for couples coping with breast cancer appear to be effective for women and their partners. However, further studies are needed to evaluate the efficacy of couple-based interventions and to identify for whom and how they are more effective.

General trends in baseline and intervention stages were compared. Individual interventions appeared to help survivors lower their high fear of cancer recurrence (FCR) and cancer-specific distress. Qualitative exit interviews conducted with study participants showed that the intervention was acceptable and satisfactory. This clinical intervention allows researchers to systematically focus on evidence-based treatments for managing FCR and displays treatment options availability in different therapeutic modalities. However, further research is needed to identify active therapeutic ingredients and mechanisms of change in the intervention. Overall, intervention studies suggested it is possible to help cancer survivors manage their FCR.

Nine studies referenced the conceptual background supporting the investigation. Five of those were based on the Transactional Model of Stress and Coping $g^{(9,38,40,59-60,65)}$ and two were based on the Social Cognitive Theory ${ }^{(38,40,68)}$. Other theoretical contributions included the Adaptation Model of Nursing ${ }^{(61,69)}$, the Crisis Intervention Model ${ }^{(60,70)}$, the Socialcognitive processing model of emotional adjustment to cancer ${ }^{(43,71)}$, the Client-centred therapy ${ }^{(46,72)}$, and the Salutogenic mode ${ }^{(46-73)}$, among others, each referenced only once.
The Cognitive-behavioral therapy ( $\mathrm{n}=9)$ was most frequent intervention used by nurses to foster cancer psychological adaptation, followed by the Educational therapy $(\mathrm{n}=6)$ and Counseling $(\mathrm{n}=6)$. Nursing psychotherapy intervention $(\mathrm{n}=2)$, Psychosocial intervention ( $n=2)$, Psychological intervention $(\mathrm{n}=1)$, Psychoeducation $(\mathrm{n}=1)$ and Supportive therapy $(\mathrm{n}=2)$ were also used.

The specific procedures used to operationalise nursing interventions were diversified. In the literature retrieved, 
telephone ( $\mathrm{n}=9)$ and face-to-face $(\mathrm{n}=8)$ interventions were the most common strategies to promote cancer psychological adaptation, but references were also found to the use of videotapes, multimedia or internet $(n=5)$, group meetings $(\mathrm{n}=3)$ and booklets $(\mathrm{n}=3)$.

In an RCT study ${ }^{(47)}$, which concluded that the effectiveness of couple coping interventions is superior to individual interventions in fostering cancer psychological adaptation, the intervention target was investigated. It was observed that most interventions were performed individually on the cancer patient $(n=11)$, an appreciable number of studies $(n=6)$ had the couple as the intervention target and a smaller number of studies $(\mathrm{n}=3)$ considered the dyad patient/caregiver ${ }^{(47)}$.

Intervention programs' length, including number of weeks and duration of each session, was also analysed. It was noted that most interventions were developed between 5 to 10 weeks $(n=11)$, included 3 to 6 sessions $(n=12)$ and lasted up to 60 minutes $(n=7)$. None of the intervention programs analysed had a duration inferior to five weeks, four had a duration of 11 to 26 weeks and only two had a duration superior to 27 weeks. Regarding the number of sessions, only one program had less than three sessions, with four programs comprising seven or more sessions. Three intervention programs lasted between 61 and 120 minutes and only one program lasted longer than 120 minutes.

An analysis of instruments used in intervention programs was performed to investigate how results of these interventions were evaluated. Despite a wide range of outcome measures employed, three were mainly identified: one (assumed as a primary outcome) focused on adjustment and coping and two (considered as secondary outcomes) focused on stress, anxiety, fear of recurrence and QoL.

A group of studies were found considering assessment of patient adjustment and coping through: Brief COPE Inventory, assessing a broad range of coping responses ${ }^{(38-39,49,59)}$; Ways of Coping Checklist, analyzing individuals' thoughts and actions to deal with specific stressful events ${ }^{(37)}$; Mini/Mental Adjustment to Cancer Scale, assessing psychological response and cancer coping styles ${ }^{(62,64)}$; Psychosocial Adjustment to Illness Scale, assessing psychological and social adjustment of medical patients ${ }^{(54,60)}$; Coping Strategies Questionnaire ${ }^{(51)}$; Profile of Adaptation to Life Clinical Scale ${ }^{(6)}$; Revised/Dyadic Adjustment Scale, widely used as an indicator of the quality of marital relationship and adjustment ${ }^{(37-39)}$; and Dyadic Coping Inventory ${ }^{(38)}$.

Instruments employed to assess stress, anxiety and fear of cancer recurrence included: Positive and Negative Affect Schedule, which comprises two mood scales - one measuring positive affect and the other, negative affect ${ }^{(43,65)}$; Hospital Anxiety and Depression Scale, which simultaneously measures depression and anxiety ${ }^{(38-39,51,63)}$; Brief Symptom Inventory, which evaluates psychological distress and psychiatric disorders ${ }^{(41,48,64)}$; Distress Thermometer, an efficient and rapid screening tool for evaluating distress in cancer populations ${ }^{(38,48-49)}$; Revised Impact of Event Scale, measuring stress reactions after traumatic events ${ }^{(39,41,65)}$; Trait Anxiety Inventory ${ }^{(37,51)}$; and Fear of Cancer Recurrence Inventory ${ }^{(67)}$.
To assess the disease impact on patients and their QoL, the European Organisation for Research and Treatment of Cancer quality of life core questionnaire - EORTC QLQC30 ${ }^{(48,54,62)}$ and the Functional Assessment of Cancer Therapy scale $^{(40,49,59,65)}$ were employed.

Some of the studies included in this review performed a feasibility assessment, i.e. analysed the extent to which an activity or intervention is practical or viable in a particular context or situation.

One of the studies included in this review ${ }^{(37)}$, which aimed to develop and evaluate the feasibility of a brief intervention to reduce the incidence of psychosexual morbidity within the dyad secondary to breast cancer diagnosis and treatment, was the only study which proposed to analyse feasibility. This study accessed feasibility and acceptability by participants of an intervention addressing intimacy and sexuality issues when coping with breast cancer and revealed that $98 \%$ of participants completed all intervention sessions and reported very positive critical evaluations of the same ${ }^{(37)}$.

An RCT protocol ${ }^{(38)}$ was developed to plan the testing of a multimedia, self-directed, coping skills training intervention for patients with cancer and their partners. The areas identified in that study as requiring greater inclusion in interventions are strategies for communicating with healthcare professionals, addressing communication difficulties between partners, dealing with emotional reactions such as fear, uncertainty, anxiety and depression in both partners and learning new skills to overcome a lack of effective coping skills. In addition, other studies ${ }^{(51,67)}$ collected participants' opinions about interventions of this type, showing that it was considered beneficial.

To analyze the effectiveness (i.e. the extent to which an intervention achieves the intended result or outcome) of studies included, only evidence level I studies were considered, i.e. those with experimental designs according to JBI Levels of Evidence: Systematic Review of Randomized Controlled Trials (RCTs), Systematic Review of RCTs and other study designs, RCTs and Pseudo-RCTs ${ }^{(74)}$.

In 2012 an RCT study ${ }^{(64)}$ reported that the intervention group displayed a significantly greater fighting spirit compared with the control group and demonstrated the potential efficacy of internet-based programs, while also highlighting their limitations. In the same year, other $\mathrm{RCT}^{(60)}$ was developed to examine the physical, emotional and social adjustment experienced by women with early-stage breast cancer who received psycho education interventions and demonstrated improvements in their overall health, psychological well-being and social adjustment.

In 2013, was showed that the intervention group which has been submitted to a multimodal comprehensive coping strategy program displayed significant improvements in overall QoL versus the control group and evidenced the potential of a self-management plan for breast cancer survivors ${ }^{(51)}$. Another study ${ }^{(59)}$ also reported greater improvements in coping, self-efficacy, social QoL and caregivers' emotional QoL in the intervention group.

Authors from other study ${ }^{(65)}$ found that the main intervention effect was a significant self-efficacy in coping with cancer 
and negative mood, also demonstrating that the intervention was effective in helping breast cancer patients to be more confident in their ability to cope with stress. In another RCT study conducted in Australia ${ }^{(41)}$, the authors suggested that a large number of distressed cancer patients and caregivers may significantly benefit from a single nursing psycho education intervention session, having their distress outcomes decreased and positive adjustments increased over the course of the trial. The results of a study ${ }^{(62)}$ that evaluated the effectiveness of a self-guided Web-based cognitive behavior therapy (CBT) intervention showed significant main effects for cancer-related distress, global QoL, physical function, role function, social function, anxious preoccupation and maladaptive coping.

Results from systematic reviews ${ }^{(55)}$ are equally important to analyse because showed the effectiveness of psychological interventions in improving 12-month survival and reducing psychological symptoms in metastatic breast cancer patients.

Another review study ${ }^{(57)}$ showed that psychosocial interventions aiming to improve $\mathrm{QoL}$, self-efficacy and knowledge and reducing distress, uncertainty and depression in men with prostate cancer produced only minor QoL improvements by the end of the study. Additionally, this study found no clear evidence that these interventions improved self-efficacy or reduced uncertainty, distress or depression. In line with this, authors from a different review ${ }^{(58)}$ also reported the lack of evidence to state that psychosocial interventions promote global QoL for patients with head and neck cancer at the end of the intervention.

However, findings from previous studies have been refuted ${ }^{(4)}$. The use of psychosocial interventions in cancer patients is supported by studies showing the relevance of cognitive, behavioural and social factors in facilitating adaptation throughout active treatment and cancer survivorship ${ }^{(4)}$. Furthermore, psychosocial interventions may improve psychological adaptation, stress and adversity response and psychological adaptation by reducing negative affect and social disruption and increasing positive affect and $\mathrm{QoL}^{(4)}$. The results of Portuguese study ${ }^{(66)}$ showed an improvement in QoL, psychological distress, relationship functioning, and physical symptoms for both members of couples coping with breast cancer, supporting psychological interventions. Lastly, other investigators ${ }^{(56)}$ found that CBT had a positive impact on psychological outcomes, particularly anxiety, depression and mood disturbance.

Although a large number of studies mention the importance of analysing interventions' cost and cost-effectiveness, none did so. Only the authors of a study mentioned that the SurvivorCare ${ }^{(48)}$ has the potential to considerably reduce individual suffering, but it is also likely to reduce the cost of health provision to this group of patients, because failing to meet patients' psychological and supportive care needs results in longer hospital stays and higher medical costs. Similarly, a developed protocol ${ }^{(38)}$ stated that the intervention cost-effectiveness would be directly assessed in the trial, but no results are known to date.

\section{DISCUSSION}

The majority of studies included in this review were performed by nurses and based their interventions on the theorical contribution by Transactional Model of Stress and Coping ${ }^{(9)}$. Although this model is derived from the principles of health psychology, and considering that autonomous nursing interventions should be based on nursing theories and expertise, this model is frequently used by oncological nurses due to its positive effect on cancer patients' psychological health, especially when the intervention focus is illness adaptation, stress management and coping mechanisms ${ }^{(75-81)}$. Nurses concerns with disease adaptation are not confined to the Psychology's body of evidence, as evidenced by use of the Adaptation Model of Nursing ${ }^{(82-83)}$ as theoretical framework for one of the studies included in this review.

Although many programs focusing oncological disease adaptation used Educational therapy and Counselling as interventional strategies, most of them used the Cognitivebehavioral therapy. Use of this technique by specialist nurses is very frequent, as exemplified in several studies, which investigated the impact of home-based exercises and cognitive behavioral therapy led by nurses on reducing cancer fatigue during and after chemotherapy ${ }^{(84)}$; which used cognitivebehavioural therapy to improve mental health of women with breast cancer ${ }^{(85)}$, and, which relied on cognitive-behavioural therapy for insomnia outcomes in women after primary breast cancer treatment ${ }^{(86)}$.

The interest in using cognitive behavioral therapy in cancer care setting has expanded, as it has been shown to effectively provide a quick and accessible way to address psychological distress and improve QoL when compared with other interventions ${ }^{(87-88)}$. Despite initially brought forward to handle depression ${ }^{(89)}$, a number of studies have consistently evidenced the applicability of Cognitive-behavioral therapy in other settings, including anxiety and symptom management and coping approach.

Regarding the content and effectiveness of programs selected in studies retrieved, it becomes clear that nurses are in a privileged position to support psychological adaptation in cancer patients through interventions promoting and training coping ability, psychological adjustment, disease self-management, and reduction of anxiety and fear of cancer recurrence $^{(37,41,44,46-48,61,67)}$.

When comparing results from our review with other studies, similar conclusions can be withdrawn. This becomes evident from a study which focusing a psychoeducational nursing intervention program to enhance coping ${ }^{(90)}$, and from another study which focusing a comprehensive coping strategy program to improve disease self-management and reduce treatment anxiety and side effects (mainly nausea and fatigue) ${ }^{(91)}$.

Results from nursing interventions are consistent with those reported in most studies found in literature. A review with meta-analysis, with the objective of perceiving the effect of psychological interventions on anxiety in cancer patients, concluded that interventions targeting individuals at risk or suffering from psychological distress have relevant clinical effects ${ }^{(92)}$. Similarly, a study reported that nurse-led cognitive behavioural guidance is effective in reducing anxiety and depression in cancer patients ${ }^{(93)}$, and ohter study showed that telephone psychosocial nursing interventions are effective in 
reducing anxiety and improving psychological QoL in breast cancer patients and their partners ${ }^{(94)}$.

An author supported the concept that oncological nurses have a fundamental role in the development of a symptom self-management plan $^{(95)}$. According to this author ${ }^{(95)}$, individualized and directed interventions are able to foster specific behaviours that cancer patients can use to recognize, prevent, reduce or mitigate the unpleasant symptoms associated with the disease and its treatment in the short-, medium- or long-term. Evidence from the literature shows that self-management symptoms can help prevent or considerably reduce disease-related suffering and hence improve the way cancer patients adjust to their new condition.

Nursing interventions developed to promote psychological adaptation are, nevertheless, wide-ranging. As observed in this review, also other studies approach adaptation indirectly, focusing on self-efficacy promotion and on mitigation the fear of cancer recurrence ${ }^{(96-98)}$.

Several authors understand that the process of psychological adaptation to oncological disease should be the main concern of nurses at every single stage of the disease and not be neglected in the survival phase, in which it is still necessary ${ }^{(60,99-100)}$. Similarly, same authors recognized the psychological adaptation process as a central, continued and dynamic element of cancer survivorship ${ }^{(101)}$.

One limitation of this work is the inclusion of English, Spanish and Portuguese studies only. Additionally, due to the multiplicity of methodological types, the quality of studies included was not evaluated (because this is not a JBI requirement for scoping reviews); other research strategies were not tested, as results would potentially be the same; and only twelve databases were considered, as the authors believed they would be the most relevant for the subject under analysis. As its main strength, the present work scrutinized and synthesized results from recent publications regarding nursing interventions directed at fostering cancer psychological adaptation in all disease stages and most tumor types.

\section{CONCLUSION}

According to scientific literature, cancer adaptation remains a complex, multidimensional and multifactorial phenomenon. Researchers in the field have revealed a keen interest in understanding the relation between psychological, emotional, familiar and social aspects of the adaptation process with patients' functional ability, well-being and QoL. It is evident that well-adapted individuals, endowed with capacitation tools to face and cope with changes caused by cancer diagnosis, treatment and living with the disease, are happier, healthier and have a better overall QoL.

It is vital that nurses, especially Oncology specialists, endow cancer patients and their families with the ability to develop coping strategies for the challenges of the disease and its consequences.

This review allowed to identify nursing programs that promote adaptive responses in adult cancer patients, to identify vulnerabilities and difficulties related to nurses' interventions and to recognize the need to produce more knowledge supporting the effectiveness and cost-effectiveness of nursing interventions, an often overlooked aspect of intervention research.

The present review is relevant, for it provides detailed information about nursing interventions in promoting cancer psychological adaptation, regardless of disease stage and tumor type. This work provides oncology investigator and/ or nurse specialists with satisfactory evidence to support decision making while planning interventions, recognize difficulties and weaknesses intrinsic to nursing interventions in the area and recognize the need to increase knowledge that secures the effectiveness and economic impact of nursing interventions.

Overall, this review is very relevant for increasing nursing knowledge due to four main reasons: i) it provides evidence that coping strategies need to be implemented to deal with a variety of anxiety factors and enhance effective psychological adaptation; ii) it recommends psychological adaptation (particularly in the context of coping and anxiety) should be the main concern of nurses at every single stage of the disease and not be neglected in the survival phase; iii) it propose the idea that specialized oncology nurses are in a prime position to support cancer patients and their intervention appears to be effective in promoting cancer psychological adaptation; iv) it create awareness the adaptation changes have the potential to influence tumor growth, functional capacity, well-being and QoL.

\section{RESUMO}

Objetivo: Mapear as evidências científicas publicadas na literatura sobre estratégias e programas de intervenção de enfermagem voltados para o apoio à adaptação psicológica em pacientes adultos com câncer. Método: Foi realizada uma revisão de escopo com base na Metodologia do Manual dos Revisores 2015 do Joanna Briggs Institute para revisões de escopo JBI. Doze bancos de dados foram pesquisados entre $1^{\circ}$ de janeiro de 2012 e 31 de janeiro de 2019. Resultados: De 2.203 estudos, 32 foram incluídos. As evidências foram agrupadas em cinco temas: procedimentos, medidas de avaliação de resultados, viabilidade de enfermagem, eficácia e custoefetividade. A terapia cognitivo-comportamental foi a intervenção mais frequente. Essas intervenções foram desenvolvidas entre 5 a 10 semanas, incluíram 3 a 6 sessões e duraram até 60 minutos. Ápesar de uma ampla gama de medidas de resultados empregadas, três áreas principais foram identificadas: ajuste e enfrentamento; estresse, ansiedade e medo de recorrência; e qualidade de vida. Conclusão: Esta revisão permitiu identificar estratégias de enfermagem para apoiar a adaptação psicológica em pacientes adultos com câncer, avaliar vulnerabilidades e dificuldades relacionadas às intervenções de enfermagem e reconhecer a necessidade de maiores esclarecimentos sobre a eficácia e custo-efetividade.

\section{DESCRITORES}

Neoplasias; Enfermagem Oncológica; Adaptação Psicológica; Revisão.

\section{RESUMEN}

Objetivo: Mapear la evidencia científica publicada en la literatura sobre estrategias y programas de intervención de enfermería dirigidos a apoyar la adaptación psicológica en pacientes adultos con cáncer. Método: Se realizó una revisión de alcance basada en la metodología 
2015 del Manual de revisores del Joanna Briggs Institute para revisiones de alcance del JBI. Se realizaron búsquedas en doce bases de datos entre el 1 de enero de 2012 y el 31 de enero de 2019. Resultados: De 2203 estudios, se incluyeron 32. La evidencia se agrupó en cinco temas: procedimientos, medidas de evaluación de resultados, viabilidad de enfermería, efectividad y costo-efectividad. La terapia cognitivo-conductual fue la intervención más frecuente. Estas intervenciones se desarrollaron entre 5 y 10 semanas, incluyeron de 3 a 6 sesiones y duraron hasta 60 minutos. A pesar de una amplia gama de medidas de resultado empleadas, se identificaron tres áreas principales, ajuste y afrontamiento; estrés, ansiedad y miedo a la recurrencia; y calidad de vida. Conclusión: Esta revisión permitió identificar estrategias de enfermería para apoyar la adaptación psicológica en pacientes adultos con cáncer, evaluar vulnerabilidades y dificultades relacionadas con las intervenciones de enfermería y reconocer la necesidad de mayores conocimientos sobre la efectividad y la rentabilidad.

\section{DESCRIPTORES}

Neoplasias; Enfermería Oncológica; Adaptación Psicológica; Revisión.

\section{REFERENCES}

1. Stanton AL, Revenson TA, Tennen H. Health psychology: psychological adjustment to chronic disease. Annu Rev Psychol. 2007;58:56592. doi: https://doi.org/10.1146/annurev.psych.58.110405.085615

2. Hoyt MA, Stanton AL. Adjustment to chronic illness. In: Baum A, Revenson TA, Singer J, editors. Handbook of health psychology. New York: Psychology Press; 2012. p. 219-46.

3. Brennan J. Adjustment to cancer: coping or personal transition? Psychooncology. 2001;10:1-18. doi: https://doi.org/10.1002/1099 1611(200101/02)10:1<1::AID-PON484>3.0.CO;2-T

4. Antoni MH. Psychosocial intervention effects on adaptation, disease course and biobehavioral processes in cancer. Brain Behav Immun. 2013;30 Suppl:S88-98. doi: https://doi.org/10.1016/j.bbi.2012.05.009

5. Piaget J. La psychologie de l'intelligence. Paris: Armand Colin; 1967.

6. Muchielli A, Muchielli R. Lexique de psychologie. Paris: Entreprise Moderne D’Édition; 1969.

7. Selye H. Stress without distress. Philadelphia: Lippincott; 1974.

8. Taylor S. Adjustment to threatening events: a theory of cognitive adaptation. Am Psychol. 1983;38:1161-73. doi: http://dx.doi. org/10.1037/0003-066X.38.11.1161

9. Lazarus RS, Folkman S. Stress, appraisal and coping. New York: Springer; 1984.

10. Greer S, Watson M. Mental adjustment to cancer: its measurement and prognostic importance. Cancer Surv. 1987;6(3):439-53.

11. Massie MJ, Holland JC. Overview of normal reactions and prevalence of psychiatric disorders. In: Holland JC, Rowland JH, editors. Handbook of psychooncology. New York: Oxford University Press; 1989. p. 273-82.

12. Watson D, Greer S, Pruyn J, Van Den Borne B. Locus of control and adjustment to cancer. Psychol Rep. 1990;66(1):39-48. doi: http:// doi.org/10.2466/pr0.1990.66.1.39

13. Rowland JH. Intrapersonal resources: coping. In: Holland JC, Rowland JH, editors. Handbook of psychooncology. New York: Oxford University Press. 1990. p. 44-54.

14. Mães S, Leventhal H, Ridder DTD. Coping with chronic diseases. In: Zeidner M, Endler NS, editors. Handbook of coping: theory, research, applications. New York: John Wiley \& Sons; 1996. p. 434-51.

15. Bandura A. The primacy of self-regulation in health promotion. Appl Psychol Int Rev. 2005;54(2):245-54. doi: https://doi.org/10.1111/ j.1464-0597.2005.00208.x

16. Ridder D, Geenen R, Kuijer R, van Middendorp H. Psychological adjustment to chronic disease. Lancet. 2008;372(9634):246-55. doi: https://doi.org/10.1016/S0140-6736(08)61078-8

17. Boinon D, Sultan S, Charles C, Stulz A, Guillemeau C, Delaloge S, et al. Changes in psychological adjustment over the course of treatment for breast cancer: the predictive role of social sharing and social support. Psycho-Oncol. 2014;23(3):291-8. doi: https://doi.org/10.1002/ pon. 3420

18. Aapro M. Too much: the paradox of cure. Oncol Pract. 1997;3:3-5.

19. Rowland J, Baker F. Introduction: resilience of cancer survivors across the lifespan. Cancer. 2005;104 Suppl 11:S2543-8. doi: https:// doi.org/10.1002/cncr.21487

20. Vachon M. Psychosocial distress and coping after cancer treatment. Cancer Nurs. 2006;29 Suppl 2:S26-31. doi: https://doi. org/10.1097/00000446-200603003-00011

21. McCorkle R, Ercolano S, Lazenby M, Schulman-Green D, Schilling LS, Lorig K, et al. Self-management: Enabling and empowering patients living with cancer as a chronic illness. CA Cancer J Clin. 2011;61(1):50-62. doi: https://doi.org/10.3322/caac.20093

22. Aziz NM. Cancer survivorship research: challenge and opportunity. J Nutr. 2002;132(11):3494-503. doi: https://doi.org/10.1093/ jn/132.11.3494S

23. Lazarus RS, Folkman S. If it changes it must be a process: study of emotion and coping during three stages of a college examination. J Pers Soc Psychol. 1985;48(1):150-70.

24. Sharpe L, Curran L. Understanding the process of adjustment to illness. Soc Sci Med. 2006;62(5):1153-66. doi: https://doi.org/10.1016/j. socscimed.2005.07.010

25. Simonet G. The concept of adaptation: interdisciplinary scope and involvement in climate change. SAPIENS. 2010;3(1):1-9.

26. Folkman S, Moskowitz JT. Coping: pitfalls and promise. Annu Rev Psychol. 2004;55(1):745-74. doi: https://doi.org/10.1146/annurev. psych.55.090902.141456 
27. Stanton AL, Revenson TA. Adjustment to chronic disease: progress and promise in research. In: Friedman HS, Silver RC, editors. Foundations of health psychology. New York: Oxford University Press. 2007. p.203-33.

28. Link LB, Robbins L, Mancuso CA, Charlson ME. How do cancer patients choose their coping strategies? A qualitative study. Patient Educ Couns. 2005;58(1):96-103. doi: https://doi.org/10.1016/j.pec.2004.07.007

29. Roesch SC, Adams L, Hines A, Palmores A, Vyas P, Tran C, et al. Coping with prostate cancer: a meta-analytic review. J Behav Med. 2005;28(3):281-93. doi: https://doi.org/10.1007/s10865-005-4664-z

30. Henderson VP, Clemow L, Massion AO, Hurley TG, Druker S, Hébert JR. The effects of mindfulness-based stress reduction on psychosocial outcomes and quality of life in early-stage breast cancer patients: a randomized trial. Breast Cancer Res Treat. 2012;131(1):99-109. doi: https://doi.org/10.1007/s10549-011-1738-1

31. Silva SM, Crespo C, Canavarro MC. Pathways for psychological adjustment in breast cancer: a longitudinal study on coping strategies and posttraumatic growth. Psychol Health. 2012;27(11):1323-41. doi: https://doi.org/10.1080/08870446.2012.676644

32. Hulbert-Williams NJ, Hulbert-Williams L, Morrison V, Neal RD, Wilkinson C. The mini-mental adjustment to cancer scale: re-analysis of its psychometric properties in a sample of 160 mixed cancer patients. Psycho-Oncol. 2012;21(7):792-7. doi: https://doi.org/10.1002/ pon. 1994

33. Kvillemo P, Bränström R. Coping with breast cancer: a meta-analysis. PLoS One 2014;9(11):1-26. doi: https://doi.org/10.1371/journal. pone. 0112733

34. Craig P, Dieppe P, Macyntire S, Michie S, Nazareth I, Petticrew M. Developing and evaluating complex interventions: the new medical research council guidance. Int J Nurs Stud. 2013;337:a1655. doi: https://doi.org/10.1136/bmj.a1655

35. Peters MDJ, Godfrey C, McInerney P, Baldini Soares C, Khalil H, Parker D. Scoping reviews. In: Aromataris E, Munn Z, editors. Joanna Briggs Institute reviewer's manual. Adelaide: JBI; 2017.

36. Tricco AC, Lillie E, Zarin W, O'Brien KK, Colquhoun H, Levac D, et al. PRISMA extension for scoping reviews (PRISMA-ScR): checklist and explanation. Ann Intern Med. 2018,169(7):467-73. doi: https://doi.org/10.7326/M18-0850

37. Decker CL, Pais S, Miller KD, Goulet R, Fifea BL. A brief intervention to minimize psychosexual morbidity in dyads coping with breast cancer. Oncol Nurs Forum. 2012;39(2):176-85. doi: https://doi.org/10.1188/12.ONF.176-185

38. Lambert SD, Girgis A, McElduff P, Turner J, Levesque JV, Kayser K, et al. A parallel-group, randomized controlled trial of a multimedia, self-directed, coping skills training intervention for patients with cancer and their partners: design and rationale. BMJ Open. 2013;3(7):113. doi: https://doi.org/10.1136/bmjopen-2013-003337

39. Lambert SD, McElduff P, Girgis A, Levesque JV, Regan TW, Turner J, et al. A pilot, multisite, randomized controlled trial of a self-directed coping skills training intervention for couples facing prostate cancer: accrual, retention, and data collection issues. Support Care Cancer. 2016;24(2):711-22. doi: https://doi.org/10.1007/s00520-015-2833-3

40. McCaughan E, Prue G, McSorley O, Northouse L, Schafenacker A, Parahoo K. A randomized controlled trial of a self-management psychosocial intervention for men with prostate cancer and their partners: a study protocol. J Adv Nurs. 2013;69(11):2572-83. doi: https://doi.org/10.1111/jan.12132

41. Chambers SK, Girgis A, Occhipinti S, Hutchison S, Turner J, McDowell M, et al. A randomized trial comparing two low-intensity psychological interventions for distressed patients with cancer and their caregivers. Oncol Nurs Forum. 2014;41(4):256-66. doi: https:// doi.org/10.1188/14.ONF.E256-E266

42. Isardas G, Holle MN. Breast cancer: psychological adjustment. CINAHL Nursing Guide; 2017.

43. Dorros SM, Segrin C, Badger TA. Cancer survivors' and partners' key concerns and quality of life. Health Psychol Rev. 2017;32(11):140727. doi: https://doi.org/10.1080/08870446.2017.1338345

44. Von AhD, Storey S, Jansen CE, Allen DH. Coping strategies and interventions for cognitive changes in patients with cancer. Semin Oncol Nurs. 2013;29(4):288-99. doi: https://doi.org/10.1016/j.soncn.2013.08.009

45. Horii N, Maekawa A. Development of a nursing model for life adjustment in patients with lung cancer in Japan. Nurs Health Sci. 2013;15(3):300-8. doi: https://doi.org/10.1111/nhs.12033

46. Sekse RJT, Blaaka G, Buestad I, Tengesdal E, Paulsen A, Vika M. Education and counselling group intervention for women treated for gynaecological cancer: does it help? Scand J Caring Sci. 2014;28(1):112-21. doi: https://doi.org/10.1111/scs.12024

47. Chen HL, Liu K, You QS. Effects of couple based coping intervention on self-efficacy and quality of life in patients with resected lung cancer. Patient Educ Couns. 2017;100(12):2297-302. doi: https://doi.org/10.1016/j.pec.2017.07.002

48. Jefford M, Aranda S, Gough K, Lotfi-Jam K, Butow P, Krishnasamy M, et al. Evaluating a nurse-led survivorship care package (SurvivorCare) for bowel cancer survivors: study protocol for a randomized controlled trial. Trials. 2013;14(1):260. doi: https://doi.org/10.1186/17456215-14-260

49. Smith SK, O'Donnell JD, Abernethy AP, MacDermott K, Staley T, Samsa GP. Evaluation of Pillars4life: a virtual coping skills program for cancer survivors. Psycho-Oncol. 2015;24(11):1407-15. doi: https://doi.org/10.1002/pon.3750

50. Whitehead L, Walker DK. Home-based multidimensional survivorship programmes for breast cancer survivors. Int J Nurs Pract. 2019;25(6):e12756. doi: 10.1111/ijn.12756

51. Gaston-Johansson F, Fall-Dickson JM, Nanda JP, Sarenmalm EK, Browall M, Goldstein N. Long-term effect of the self-management comprehensive coping strategy program on quality of life in patients with breast cancer treated with high-dose chemotherapy. PsychoOncol. 2013;22(3):530-9. doi: https://doi.org/10.1002/pon.3031

52. Thomas LPM, Meier EA, Irwin SA. Meaning-centered psychotherapy: a form of psychotherapy for patients with cancer. Curr Psychiatry Rep. 2014;16(10):488-8. doi: https://doi.org/10.1007/s11920-014-0488-2

53. Scott DA, Mills M, Black A, Cantwell M, Campbell A, Cardwell CR, et al. Multidimensional rehabilitation programmes for adult cancer survivors. Cochrane Database Syst Rev. 2013;(3):CD007730. doi: https://doi.org/10.1002/14651858.CD007730.pub2 
54. Leeuw J, Prins JB, Teerenstra S, Merkx MAW, Marres HAM, van Achterberg T. Nurse-led follow-up care for head and neck cancer patients: a quasi-experimental prospective trial. Support Care Cancer. 2013;21(2):537-47. doi: https://doi.org/10.1007/s00520-012-1553-1

55. Mustafa M, Carson-Stevens A, Gillespie D, Edwards AG. Psychological interventions for women with metastatic breast cancer. Cochrane Database Syst Rev. 2013;(6):CD004253. doi: https://doi.org/10.1002/14651858.CD004253.pub4

56. Jassim GA, Whitford DL, Hickey A, Carter B. Psychological interventions for women with non-metastatic breast cancer. Cochrane Database Syst Rev. 2015;(5):CD008729. doi: https://doi.org/10.1002/14651858.CD008729.pub2

57. Parahoo K, McDonough S, McCaughan E, Noyes J, Semple C, Halstead EJ, et al. Psychosocial interventions for men with prostate cancer. Cochrane Database Syst Rev. 2013;(12):CD008529. doi: https://doi.org/10.1002/14651858.CD008529.pub3

58. Semple C, Parahoo K, Norman A, McCaughan E, Humphris G, Mills M. Psychosocial interventions for patients with head and neck cancer. Cochrane Database Syst Rev. 2013;(7):CD009441. doi: https://doi.org/10.1002/14651858.CD009441.pub2

59. Northouse LL, Mood DW, Schafenacker A, Kalemkerian G, Zalupski M, LoRusso P, et al. Randomized clinical trial of a brief and extensive dyadic intervention for advanced cancer patients and their family caregivers. Psycho-Oncol. 2013;22(3):555-63. doi: https:// doi.org/10.1002/pon.3036

60. Sherman DW, Haber J, Hoskins CN, Budin WC, Maislin G, Shukla S, et al. The effects of psychoeducation and telephone counseling on the adjustment of women with early-stage breast cancer. Appl Nurs Res. 2012;25(1):3-16. doi: https://doi.org/10.1016/j.apnr.2009.10.003

61. Salgado A. Intervenções de enfermagem promotoras da adaptação à doença oncológica mamária [Internet]. Lisboa: ICBAS; 2013 [cited 2019 Dec 17]. Available from: https://repositorio-aberto.up.pt/handle/10216/70855?locale=pt

62. Beatty L, Koczwara B, Wade T. Evaluating the efficacy of a self-guided Web-based CBT intervention for reducing cancer-distress: a randomized controlled trial. Support Care Cancer. 2016;24(3):1043-51. doi: https://doi.org/10.1007/s00520-015-2867-6

63. García A, Hurtado A, Aranda B. Eficacia de la terapia cognitivo conductual en mujeres con cáncer de mama. Psicooncología. 2015;12(1):129-40. doi: https://doi.org/10.5209/rev_PSIC.2015.v12.n1.48908

64. David N, Schlenker P, Prudlo U, Larbig W. Internet-based program for coping with cancer: a randomized controlled trial with hematologic cancer patients. Psycho-Oncol. 2012;22(5):1064-72. doi: https://doi.org/10.1002/pon.3104

65. Carpenter KM, Stoner SA, Schmitz K, McGregor BA, Doorenbos AZ. An Online Stress Manage Workbook Breast Cancer. J Behav Med. 2014;37(3):458-68. doi: https://doi.org/10.1007/s10865-012-9481-6

66. Brandão T, Schulz MS, Matos PM. Psychological intervention with couples coping with breast cancer: a systematic review. Psychol Health. 2014;29(5):491-516. doi: https://doi.org/10.1080/08870446.2013.859257

67. Tomei C, Lebel S, Maheu C, Mutsaers B. Addressing fear of recurrence: improving psychological care in cancer survivors. Support Care Cancer. 2016;24(7):2815-8. doi: https://doi.org/10.1007/s00520-016-3103-8

68. Bandura A. Social foundations of thought and action: a social cognitive theory. Englewood Cliffs: Prentice-Hall; 1986.

69. Roy C, Andrews H. Teoria da enfermagem: o modelo de adaptação de Roy. Lisboa: Instituto Piaget; 2001.

70. Morely WE, Messick JM, Aguilera DC. Crisis: paradigms of intervention. J Psychiatr Nurs. 1967;5(6):531-44.

71. Lepore SJ. A social cognitive processing model of emotional adjustment to cancer. In: Baum A, Andersen BL, editors. Psychosocial interventions for cancer. Washington, American Psychological Association; 2001. p. 99-116.

72. Rogers CR. Client-centered therapy. Oxford: Houghton Mifflin; 1951.

73. Antonovsky, A. The salutogenic model as a theory to guide health promotion. Health Promot Int. 1996;11(1):11-8. doi: https://doi. org/10.1093/heapro/11.1.11

74. Joanna Briggs Institute. New JBI levels of evidence [Internet]. Adelaide: JBI; 2013 [cited 2019 Dec 17]. Available from: http://joannabriggs. org/assets/docs/approach/JBI-Levels-of-evidence_2014.pdf

75. Hilton BA. The relationship of uncertainty, control, commitment, and threat of recurrence to coping strategies used by women diagnosed with breast cancer. J Behav Med. 1989;12(1):39-54. doi: https://doi.org/10.1007/BF00844748

76. Balneaves LG, Long B. An embedded decisional model of stress and coping: implications for exploring treatment decision making by women with breast cancer. J Adv Nurs. 1999;30:1321-31. doi: https://doi.org/10.1046/j.1365-2648.1999.01239.x

77. Gall TL. Integrating religious resources within a general model of stress and coping: long-term adjustment to breast cancer. J Relig Health 2000;39:167-82. doi: https://doi.org/10.1023/A:1004670717144

78. Laubmeier KK, Zakowski SG, Bair JP. The role of spirituality in the psychological adjustment to cancer: a test of the transactional model of stress and coping. Int J Behav Med. 2004;11(1):48-55. doi: https://doi.org/10.1207/s15327558ijbm1101_6

79. Street A, Couper J, Love A, Bloch S, Kissane D, Street B. Psychosocial adaptation in female partners of men with prostate cancer. Eur J Cancer Care. 2010;19(2):234-242. doi: https://doi.org/10.1111/j.1365-2354.2008.01012.x

80. Drageset S, Lindstrøm TC, Underlid K. Coping with breast cancer: between diagnosis and surgery: between diagnosis and surgery. J Adv Nurs. 2010;66(1):149-58. doi: https://doi.org/10.1111/j.1365-2648.2009.05210.x

81. Taniguchi A, Mizuno M. Psychological stress and coping in recently discharged postsurgical cancer patients. Asia Pac J Oncol Nurs. 2016;3(2):176-82. doi: http://doi.org/10.4103/2347-5625.177394

82. Roy SC. Introduction to nursing: an adaptation model. $2^{\text {nd }}$ ed. Englesood Cliffis: Prentice Hall; 1984.

83. Roy SC. The Roy adaptation model. $3^{\text {th }}$ ed. New Jersey: Pearson Education; 2009.

84. Zhang Q, Li F, Zhang H, Yu X, Cong Y. Effects of nurse-led home-based exercise \& cognitive behavioral therapy on reducing cancerrelated fatigue in patients with ovarian cancer during and after chemotherapy: a randomized controlled trial. Int J Nurs Stud. 2018;78:5260. doi: http://dx.doi.org/10.1016/j.ijnurstu.2017.08.010 
85. Li-Hung L, Yu-Ping L, Cheng-I Y. Using the cognitive behavioral therapy to Improve the mental health of women with breast cancer. Hu Li Za Zhi. 2017;64(2):28-33. doi: http://dx.doi.org/10.6224/JN.000019

86. Matthews EE, Berger AM, Schmiege SJ, Cook PF, McCarthy MS, Moore CM, et al. Cognitive behavioral therapy for insomnia outcomes in women after primary breast cancer treatment: a randomized, controlled trial. Oncol Nurs Forum. 2014;41(3):241-53. doi: http://dx.doi. org/10.1188/14.ONF.41-03AP

87. Sage N, Sowden M, Chorlton E, Edeleanu A. CBT for chronic illness and palliative care: a workbook and toolkit. New York: John Wiley \& Sons; 2008.

88. Pitceathly CP, Maguire P, Fletcher I, Parle M, Tomenson B, Creed F. Can a brief psychological intervention prevent anxiety or depressive disorders in cancer patients? A randomised controlled trial. Ann Oncol. 2009;20(5):928-34. doi: http://dx.doi.org/10.1093/annonc/ $\operatorname{mdn} 708$

89. Beck AT. Depression: clinical, experimental, and theoretical aspects. New York: Harper and Row; 1967.

90. Fawzy NW. A psychoeducational nursing intervention to enhance coping and affective state in newly diagnosed malignant melanoma patients. Cancer Nurs. 1995;18(6):427-38.

91. Gaston-Johansson F, Fall-Dickson JM, Nanda J, Ohly KV, Stillman S, Krumm S, et al. The effectiveness of the comprehensive coping strategy program on clinical outcomes in breast cancer autologous bone marrow transplantation. Cancer Nurs. 2000;23(4):277-85. doi: https://doi.org/10.1097/00002820-200008000-00004

92. Sheard T, Maguire P. The effect of psychological interventions on anxiety and depression in cancer patients: results of two meta-analyses. Br J Cancer. 1999;80(11): 1770-80. doi: https://doi.org/10.1038/sj.bjc.6690596

93. Yoo MS, Lee H, Yoon JA. Effects of a cognitive-behavioral nursing intervention on anxiety and depression in women with breast cancer undergoing radiotherapy. J Korean Acad Nurs. 2009;39(2):157-65. doi: https://doi.org/10.4040/jkan.2009.39.2.157

94. Badger T, Segrin C, Dorros SM, Meek P, Lopez AM. Depression and anxiety in women with breast cancer and their partners. Nurs Res. 2007;56(1):44-53. doi: https://doi.org/10.1097/00006199-200701000-00006

95. Hoffman AJ. Enhancing self-efficacy for optimized patient outcomes through the theory of symptom self-management. Cancer Nurs. 2013;36(1):16-26. doi: https://doi.org/10.1097/NCC.0b013e31824a730a

96. Mast ME. Survivors of breast cancer: illness uncertainty, positive reappraisal, and emotional distress. Oncol Nurs Forum. 1998;25(3):555-62.

97. Custers JA, van den Berg SW, van Laarhoven HW, Bleiker EM, Gielissen MF, Prins JB. The Cancer Worry Scale: detecting fear of recurrence in breast cancer survivors. Cancer Nurs. 2014;37(1):44-50. doi: https://doi.org/10.1097/NCC.0b013e3182813a17

98. Custers JA, Gielissen MF, Janssen SH, Wilt JH, Prins JB. Fear of cancer recurrence in colorectal cancer survivors. Support Care Cancer. 2016;24(2):555-562. doi: https://doi.org/10.1007/s00520-015-2808-4

99. Livneh H. Psychosocial adaptation to chronic illness and disability: a conceptual framework. Rehabil Couns Bull. 2001;44(3):151-60. doi: https://doi.org/10.1177/003435520104400305

100. Sheldon LK, Harris D, Arcieri C. Psychosocial concerns in cancer care: the role of the oncology nurse. Clin J Oncol Nurs. 2012;16(3):3169. doi: https://doi.org/10.1188/12.CJON.316-319

101. Naus MJ, Ishler MD, Parrott CE, Kovacs SA. Cancer survivor adaptation model: conceptualizing cancer as a chronic illness. J Clin Psychol. 2009;65(12):1350-9. doi: https://doi.org/10.1002/jclp.20622 